\title{
Publication bias in clinical trials (Protocol)
}

\author{
Olsen KL, Hopewell S, Dickersin K, Clarke M, Oxman AD
}

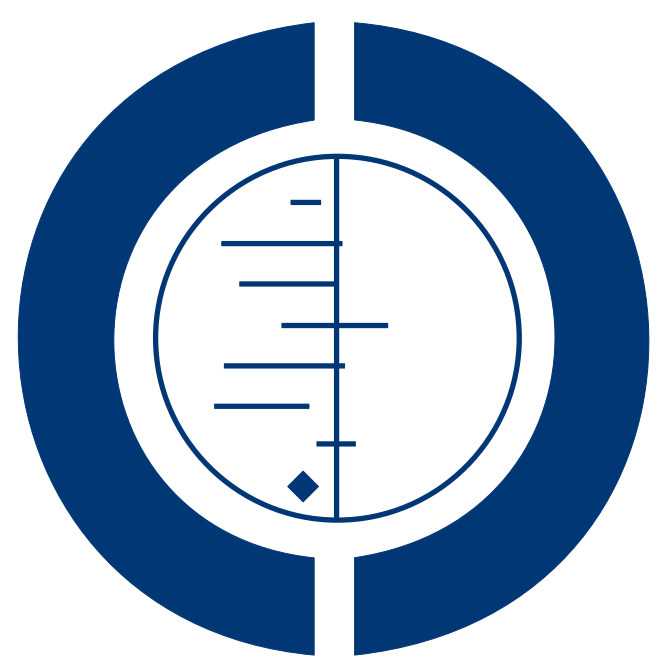

\section{THE COCHRANE COLLABORATION $^{\circledR}$}

This is a reprint of a Cochrane protocol, prepared and maintained by The Cochrane Collaboration and published in The Cochrane Library 2001, Issue 3

\author{
http://www.thecochranelibrary.com
}

\section{WILEY}




\section{TABLE OF CONTENTS}

ABSTRACT . . . . . . . . . . . . . . . . . . . . . . . . . . . . . . . . . . . . . . . 1

BACKGROUND . . . . . . . . . . . . . . . . . . . . . . . . . . . . . . . . . . . . . . 1

OBJECTIVES . . . . . . . . . . . . . . . . . . . . . . . . . . . . . . . . . . . . . . . . . . . . . . . 1

CRITERIA FOR CONSIDERING STUDIES FOR THIS REVIEW . . . . . . . . . . . . . . . . . . . . . . . 1

SEARCH METHODS FOR IDENTIFICATION OF STUDIES . . . . . . . . . . . . . . . . . . . . . . . . . . 2

METHODS OF THE REVIEW . . . . . . . . . . . . . . . . . . . . . . . . . . . . . . . . 2

POTENTIAL CONFLICT OF INTEREST . . . . . . . . . . . . . . . . . . . . . . . . . . . . . . 3

ACKNOWLEDGEMENTS . . . . . . . . . . . . . . . . . . . . . . . . . . . . . . . . . . . . 3

SOURCES OF SUPPORT . . . . . . . . . . . . . . . . . . . . . . . . . . . . . . . . . . . . . . . . . 4.4

REFERENCES . . . . . . . . . . . . . . . . . . . . . . . . . . . . . . . . . . . . . . . . 44

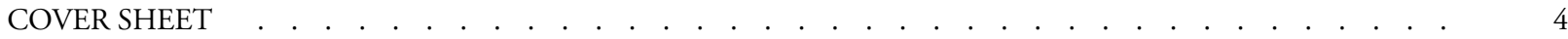

Publication bias in clinical trials (Protocol)

Copyright $\odot 2007$ The Cochrane Collaboration. Published by John Wiley \& Sons, Ltd 


\title{
Publication bias in clinical trials (Protocol)
}

\author{
Olsen KL, Hopewell S, Dickersin K, Clarke M, Oxman AD
}

This record should be cited as:

Olsen KL, Hopewell S, Dickersin K, Clarke M, Oxman AD. Publication bias in clinical trials. (Protocol) Cochrane Database of Methodology Reviews 2001, Issue 3. Art. No.: MR000006. DOI: 10.1002/14651858.MR000006.

This version first published online: 23 July 2001 in Issue 3, 2001.

Date of most recent substantive amendment: 25 May 2001

\section{A B S T R A C T}

This is the protocol for a review and there is no abstract. The objectives are as follows:

To summarise evidence of publication bias for trials of health care interventions.

\section{B A C K G R O U N D}

Completed research is frequently left unpublished (Dickersin 1990). It has been suggested that in the case of research conducted on humans, failure to publish represents scientific misconduct, since individuals who consent to participate in research, and agencies that provide funding support for investigations, do so with the understanding that the work will make a contribution to knowledge (Chalmers 1990). Clearly, knowledge that is not disseminated is not making a "contribution".

Failure to publish is not only inappropriate scientific conduct; it also influences the information available for interpretation by the scientific community. If research is left randomly unpublished, there is less information available, but that information is not necessarily biased. The tendency for investigators to submit manuscripts and of editors and reviewers to accept them, based on the strength and direction of the research findings, has been defined as publication bias (Chalmers 1990, Dickersin 1990, Dickersin 1997).

Song et al (Song 2000) also suggested that this term might include biases related to time, the type and language of publication, multiple publication and selective citation of references. They suggest that dissemination bias may be a better expression with which to replace this broad use of the term for selective publication.

Publication bias in health care has been discussed for many years (Dickersin 1987, Simes 1986, Simes 1987) and those who carry out systematic reviews need to identify as unbiased and complete a set of relevant studies as possible for inclusion in their review; otherwise the conclusions could potentially be biased and therefore may be invalid.

Three ways to minimise the effect of publication bias on a systematic review were outlined by Song et al. First, by accessing a register of prospective trials when undertaking a systematic review, secondly by seeking to detect publication bias during a review (for example by locating unpublished studies) and thirdly, by reducing the impact of publication bias by updating the review when trials become available or undertaking confirmatory large-scale trials (Song 2000).

This systematic review of methodology is the update of an original meta-analysis by one of the authors (KD), which aims to assess the evidence of publication bias in a series of cohorts of registered studies (Dickersin 1992, Dickersin 1993, Dickersin 1997, Easterbrook 1991, Stern 1996, Stern 1997).

\section{O B J E C T I VES}

To summarise evidence of publication bias for trials of health care interventions.

\section{CRITERIA FOR CONSIDERING STUDIES FOR THIS REVIEW}

\section{Types of studies}

Cohorts of trials "registered" (i.e. trials entered into a formal database or some other list such as for submissions to an Insti- 
tutional Review Board or a prospective trials register) at onset or while ongoing, but prior to the main results being known.

\section{Types of data}

Must be either a complete series of trials (e.g. all registered during a specified time period) or an unbiased sample (e.g. random sample) of trials. Studies are accepted as clinical trials if they are so defined by the study authors, involve the testing of a health care intervention and include people as participants. Studies that include nontrial research are eligible for the review, if data specifically related to clinical trials are available and are able to be analysed separately from the non-trials. If these results cannot be separated using the information in the published report, attempts will be made to obtain the data from the study investigators. The primary analysis will be based on an assessment of trials comparing a standard intervention (as defined by the trialists) versus a new or experimental intervention.

\section{Types of comparisons}

The publication rate of trials with positive findings will be compared to the publication rate of those with "negative" findings, based on the $\mathrm{p}$-value $(<0.05$ versus $>0.05$ respectively, unless otherwise specified). We will also compare the publication rate of positive versus negative trials by considering the direction of effect and the magnitude of effect. Positive findings are defined as those with statistically significant results $(\mathrm{p}<0.05)$ in favour of the experimental arm of the trial. "Negative" findings are broadly defined as those with non-statistically significant results $(p>0.05)$ or results in favour of the control arm. Statistically significant findings in favour of the control arm and non-statistically significant results will be considered separately, although "negative" findings is used to refer to both. If sufficient data are available, other factors that may be associated with failure to publish will be examined. These include study quality, number of study groups, source of funding, sample size, number of clinical centres, and investigator rank and sex.

\section{Types of outcome measures}

The primary outcomes will be publication and time from completion of research (end of data collection) to full publication in a journal. Secondary outcomes will include whether or not the study results were written up, reasons for failure to publish, publication in English versus other languages, publications indexed in MEDLINE versus non-MEDLINE, and different types of publication (e.g. grey literature, including in-house publications and theses). These outcomes will be reported by study findings (positive versus negative).

\section{SEARCH METHODS FOR IDENTIFICATIONOF STUDIES}

See: methods used in reviews.
The Cochrane Methodology Register will be searched using the index term "publication bias" including language bias and duplicate publication bias. MEDLINE and PREMEDLINE will be searched using OVID with the following terms:

\#1 Publication Bias (MeSH)

\#2 Exp. Publications (MeSH) OR publication\$ (text word) OR publishing $(\mathrm{MeSH}) \mathrm{OR}$ publish\$ (text word)

\#3 Exp. bias (epidemiology) (MeSH) OR bias (text word)

\#4 2 and 3

\#5 1 or 4

We will also search for "related articles" in MEDLINE for studies that meet the inclusion criteria. EMBASE will be searched using OVID with the following terms:

\#1 Publishing (EMTREE) OR Publishing (text word) \#2 Publication (EMTREE) OR Publication (text word)

\#3 Bias (text word)

\#4 1 or 2

\#5 3 and 4

We will search SciSearch, using articles deemed eligible for the review, to identify additional articles that cite studies that meet our inclusion criteria. Finally we will contact methodologists and authors of key studies on publication bias to try and identify further studies. We will check reference lists of relevant articles to identify references to possible relevant citations.

\section{METHODS OF THE REVIEW}

\section{Identifying studies}

One reviewer (KLO) will screen the titles and abstracts of all retrieved records to identify obvious exclusions. A second reviewer (SH) will check the exclusion of other less obvious records, before rejection. Any disagreements will be resolved through discussion. Each of the non-rejected records will be assessed by at least two of the reviewers to see if they are likely to meet the inclusion criteria and full copies of reprints will be obtained for these reports. Each of the full reports will then be assessed by at least two reviewers to determine if they meet the inclusion criteria for the review. Any disagreements will be resolved through discussion.

\section{Assessment of methodological quality}

The following criteria will be used to assess the methodological quality of included studies:

1. Was there an inception cohort?

Yes $=$ a complete sample of clinical trials registered at onset during a specified period of time

No = anything else

Unclear

2. Was there complete follow-up (after data analysis) of all of the trials in the cohort for at least four years? 
Yes $>90 \%$

No $<90 \%$

Unclear

3. Was publication ascertained through personal contact with the investigators?

Yes = personal contact with investigators, or searching the literature and personal contact with investigator

No $=$ searching the literature only

Unclear

4.Was positive and negative clearly defined and based on the primary outcome(s) as defined by the investigators (prior to obtaining results)?

Yes = clearly defined and based on primary outcomes in trial protocols

No = not clearly defined or not based on primary outcomes in trial protocols

Unclear

5.Were other possible confounders controlled for in the analysis, including: sample size, duration, multi-centre versus single centre, funding (external versus internal, industry funded versus other), and investigator rank and were trials grouped for common treatment comparisons?

Yes $=$ two or more of the above.

No $=$ one or none

Unclear

Overall quality of the studies will be summarised as "good" if all of the above criteria are met, "moderate" (all but one met) and "possibly problematic" (two or more not met).

\section{Data extraction}

Two reviewers (KLO and $\mathrm{SH}$ ) will independently extract data. Differences in data extraction will be resolved by discussion. The reviewers will contact investigators to obtain information or data needed for the review that could not be found in published reports.

Data will be extracted on the following:

$P$ value $(<0.05$ versus $>0.05)$

Direction of results (positive or negative) - as defined above

Magnitude of effect

Higher rating by the investigator on the importance of study results versus lower rating. (However defined in the included studies)

Funding mechanism (external versus internal, and industry funding versus other)

Actual sample size $(<100,100-999,>999$ or as defined in included studies)

Multi or single centre trials

Primary investigator sex (male versus female) - first author

Primary investigator rank (e.g. professor, associate professor, assistant professor, other) - first author

for the following outcomes: full publication

other types of publication (e.g. grey literature, abstract, presentation, language of publication)

We will also collect details regarding other characteristics of the types of data (countries, institutions, registers, inclusion criteria for trials), types of comparisons, outcome measures for the included studies, and means and duration of follow-up for the trials in the cohort.

\section{Data analysis}

The primary analyses will compare the publication rates and time to publication for positive and negative studies based on $\mathrm{p}$ values (or as reported by the study). Publication rates and time to publication will also be analysed for direction of effect and magnitude of effect. If possible, the analyses will be conducted using raw data in Review Manager using relative risks (MantelHaensel method). If the p-value for the chi-square test for heterogeneity is $>0.10$ we will summarise the overall relative risk using the fixed effect model (Mantel-Haensel method). If there is significant heterogeneity $(\mathrm{p}<0.10)$ we will consider the following possible explanations in subgroup analyses:

Length of follow-up

Overall quality of the studies

Adjusted for confounders

Time to publication will be analysed in the same way using weighted means. If possible, secondary analyses will compare pooled adjusted odds ratios to pooled un-adjusted odds ratios (OR) for studies for which adjusted ORs can be obtained. We will also compare time to publication controlling for confounders and time to publication without controlling for confounders. The possibility of publication bias for our included studies of publication bias will be investigated using the Funnel plot.

\section{POTENTIAL CONFLICTOF I N T ER ES T}

Kay Dickersin and Mike Clarke are involved in the development of trial registers such as the Cochrane Controlled Trials Register.

\section{ACKNOWLEDGEMENTS}

We would like to thank Gunn Vist for her editorial assistance, Peter Gøtzsche for his editorial advice and John Simes for his helpful comments. 


\section{SOURCES OF SUPPORT}

\section{External sources of support}

- No sources of support supplied

Internal sources of support

- National Institute of Public Health NORWAY

- NHS Research and Development Programme UK

- Brown University USA

\section{R E F E R E N C E S}

\author{
Additional references \\ Chalmers 1990 \\ Chalmers I. Underreporting research is scientific misconduct. JAMA \\ 1990;263:1405-8. \\ Dickersin 1987 \\ Dickersin K, Chan S, Chalmers TC, Sacks HS, Smith H. Publication \\ bias and clinical trials. Controlled Clinical Trials 1987;8:343-53. \\ Dickersin 1990 \\ Dickersin K. The existence of publication bias and risk factors for its \\ occurrence. JAMA 1990;206:1385-9. \\ Dickersin 1992 \\ Dickersin K, Min Yi, Meinert CL. Factors influencing publication \\ of research results: follow-up of applications submitted to two insti- \\ tutional review boards. JAMA 1992;263:274-8.

\section{Dickersin 1993} \\ Dickersin K, Min Yi. NIH clinical trials and publication bias. Online \\ Journal of Current Clinical Trials (serial online) 1993, (Doc-No 50): \\ 4987 words, 53 paragraphs. \\ Dickersin 1997 \\ Dickersin K. How important is publication bias? A synthesis of avail- \\ able data. AIDS Education \& Prevention 1997;9(Suppl A):15-21.
}

Easterbrook 1991

Easterbrook PJ, Berlin JA, Gopalan R, Matthews DR. Publication bias in clinical research. Lancet 1991;337:867-72.

Simes 1986

Simes RJ. Publication bias: the case for an international registry of clinical trials. Journal of Clinical Oncology 1986;4:1529-41.

Simes 1987

Simes RJ. Confronting publication bias: a cohort design for metaanalysis. Statistics in Medicine 1987;6:11-29.

Song 2000

Song F, Eastwood AJ, Gilbody S, Duley L, Sutton AJ. Publication and related biases. Health Technology Assessment 2000;4(10):1-115.

Stern 1996

Stern J. Publication bias - An Australian perspective: A review of 801 research proposals submitted to the Royal Alfred Hospital Ethics Committee between 1979 and 1988 [Masters Degree Thesis]. Sydney: University of Sydney, 1996.

Stern 1997

Stern JM, Simes RJ. Publication bias: evidence of delayed publication in a cohort study of clinical research projects. BMJ 1997;315:640-5.

\section{COVER SHEET}

Title

\section{Authors}

\section{Contribution of author(s)}

Issue protocol first published

Date of most recent amendment

Date of most recent SUBSTANTIVE amendment

What's New

Contact address
Publication bias in clinical trials

Olsen KL, Hopewell S, Dickersin K, Clarke M, Oxman AD

KD prepared the first draft of the protocol. KLO had primary responsibility for subsequent revisions of the protocol. All of the reviewers contributed to revising the protocol.

$2001 / 3$

10 May 2004

25 May 2001

Information not supplied by author

Kirsty L. Olsen

3 Burnside

Eddleston 
Peebles

EH45 8RH

UK

E-mail: kirstylolsen@yahoo.co.uk

Tel: 01721729040

DOI

Cochrane Library number

Editorial group

Editorial group code
10.1002/14651858.MR000006

MR000006

Cochrane Methodology Review Group

HM-METHOD 